Family Medicine and Community Health

\section{Upper arm length along with mid-upper arm circumference to enhance wasting prevalence estimation and diagnosis: sensitivity and specificity in 6-59-months-old children}

To cite: Barro M, Baro MD Cisse D, et al. Upper arm length along with mid-upper arm circumference to enhance wasting prevalence estimation and diagnosis: sensitivity and specificity in 6-59-months-old children. Fam Med Com Health 2021;9:e000748. doi:10.1136/ fmch-2020-000748

- Additional supplemental material is published online only. To view, please visit the journal online (http://dx.doi.org/10. 1136/fmch-2020-000748).
Check for updates

(C) Author(s) (or their employer(s)) 2021. Re-use permitted under CC BY-NC. No commercial re-use. See rights and permissions. Published by BMJ.

${ }^{1}$ Nutrition and development association, Nouakchott,

Mauritania

${ }^{2}$ Nutrition, UNICEF, Nouakchott, Mauritania

${ }^{3}$ UNICEF West and Central Africa Regional Office, Dakar, Senegal ${ }^{4}$ Ministry of Health, Nouakchott, Mauritania

${ }^{5}$ Independent Consultant in Biostatistics, Paris, France

${ }^{6}$ University of Nouakchott, Nouakchott, Mauritania

Correspondence to Mouhamed Barro; mohamed-racine-barro@ hotmail.fr

\section{ABSTRACT}

Objective To evaluate the added value of the use of upper arm length (UAL) along with mid-upper arm circumference (MUAC) to diagnose and estimate the prevalence of wasting in comparison to current WHO standard and other MUAC-based methods.

Design UAL and usual anthropometric measurements were collected during a national cross-sectional nutritional survey. Children were classified into three upper arm length groups (UALGs): UALG1, UALG2 and UALG3 according to the following UAL limits: $\leq 150,151-180$ and $\geq 181 \mathrm{~mm}$, respectively. Receiver operating characteristic curves were used to determine the best MUAC cut-off for each group using weight-for-height Z-score (WHZ) as a reference standard. Wasting prevalence, sensitivity and specificity of all diagnostic methods were compared.

Setting This study was conducted in Mauritania. Participants National representative sample of children from 6 to 59 months old.

Results In total, 12590 children were included in the study. Wasting prevalence was $16.1 \%, 5.0 \%$ and $12.5 \%$ when diagnosed by $\mathrm{WHZ}<-2$, MUAC $<125$ $\mathrm{mm}$ and MUAC-UALG methods, respectively. Using the MUAC-UALG method increased the sensitivity for wasting diagnosis from $17.98 \%$ with MUAC $<125 \mathrm{~mm}$ to $39.43 \%$ with MUAC-UALG. The specificity decreased from $97.49 \%$ with MUAC $<125 \mathrm{~mm}$ to $92.71 \%$ with MUAC-UALG. With MUAC-height Z score and MUAC $<138 \mathrm{~mm}$, sensitivity was $26.04 \%$ and $69.76 \%$ and specificity were $97.40 \%$ and $75.64 \%$ respectively.

Conclusion This alternative method using MUAC tape to measure UAL increases the wasting diagnosis accuracy and allows for a better estimation of wasting prevalence. This method could be used as a potential alternative method for quick surveys in emergency settings such as Corona virus disease 2019 context.

\section{INTRODUCTION}

Wasting is a major public health problem in low-income and middle-income countries (LMIC). The risk of death is higher in wasted children defined by a

\section{Key messages}

\section{Question}

- Can mid-upper arm circumference (MUAC)-based wasting prevalence estimation as well as diagnosis accuracy be improved by using upper arm length (UAL)?

\section{Finding}

- Yes, the use of UAL along with MUAC improves wasting prevalence estimation and wasting diagnosis in Mauritanian children.

\section{Meaning}

- It should be possible to use UAL along with MUAC for a rapid wasting prevalence estimation in the field as well as wasting management admission and discharge criteria.

weight-for-height Z-score (WHZ) below -2 , when compared with non-wasted children. ${ }^{1}$ When diagnosed with wasting, children can be treated at home. ${ }^{2}$ The earlier the child is diagnosed, the shorter the duration of the treatment. ${ }^{3}$ However, wasting screening and diagnosis has been a challenge for the entire humanitarian community. WHZ remains difficult to obtain routinely at the community level as it requires heavy equipment and welltrained staff. Mid-upper arm circumference (MUAC) is therefore preferred in the field due to its simplicity (MUAC $<115$ $\mathrm{mm}$ for severe wasting, MUAC $<125 \mathrm{~mm}$ for wasting) as per the WHO recommendations. ${ }^{1}$ However, MUAC has shown its limits for wasting diagnosis as well as prevalence estimation.

In 2019, wasting (as defined by WHZ score below -2) affected more than 47 million children under 5 years old 
world-wide. ${ }^{4}$ Although both low WHZ and MUAC are recommended for wasting diagnosis, only low WHZ is used for wasting prevalence evaluation by WHO. ${ }^{14}$ The use of current WHO's MUAC cut-off recommendation does not allow for wasting prevalence estimation with an acceptable accuracy. ${ }^{5}$

Different MUAC cut-offs have been proposed in the past decades for wasting diagnosis (also called acute malnutrition). In the 1960 s, a study based on a population of non-malnourished Polish children showed that MUAC had little or no relation to age and gender in children aged 1-5 years. ${ }^{6}$ Shakir and Morley suggested a coloured cord to measure upper-arm circumference for screening and diagnosis of wasting in children 6-59-months old. ${ }^{7}$ Children were categorised in three groups according to their MUAC: red, yellow and green for MUAC under $125 \mathrm{~mm}$, between $125 \mathrm{~mm}$ and $135 \mathrm{~mm}$, and over $135 \mathrm{~mm}$, respectively. In 1985, Lindtjorn showed that these cut-off points greatly exaggerate wasting prevalence rates and proposed new cut-off points $(110$ and $130 \mathrm{~mm}) .{ }^{8}$ Benr and Nathanail compared the WHZ $<-2$ and MUAC $<125$ $\mathrm{mm}$ methods and concluded that these two methods identify similar proportions of wasted children. ${ }^{9}$ However, beyond the cut-off point itself, the use of a single cut-off for wasting diagnosis in all children within this age range has been debated. ${ }^{10}{ }^{11}$ Indeed, MUAC has been reported to be age-specific and the use of MUAC with a single cut-off underestimates wasting in older children. ${ }^{12} 13$ To address this bias, a MUAC-based method taking into account child's age and sex has been implemented. A Z-score is assigned to each child according to their MUAC, age and sex. ${ }^{14}$ However, the difficulty of determining the children's age led to the use of another index, based on MUAC, height and sex. ${ }^{15}$ These methods certainly improve the sensitivity of wasting diagnosis but are not simple enough to be used for routine diagnoses. In fact, the determination of the children's age on the one hand and their exact height on the other hand are essential for the MUAC-age and MUAC-height indices. Due to the necessity of calculating the Z-score for each child, both methods are not really routinely used in the field.

We therefore considered an alternative method for wasting prevalence estimation, as well as wasting diagnosis with greater sensitivity and greater potential for routine use. Children's height or age is not required. The method is based on the use of MUAC in relation to child's upper arm length (UAL) which can be measured at the same time as the MUAC measurement, using the same MUAC tape. We tested this method in a nutritional survey conducted in July 2015 according to the methodology 'Standardised Monitoring and Assessment of Relief and Transitions' (SMART) in Mauritania. The current study aimed at evaluating the added value of the use of UAL along with the MUAC to diagnose and estimate the prevalence of wasting in comparison to the WHO standard as well as other MUAC based methods.

\section{METHODOLOGY \\ Data collection}

Data collected from the national SMART survey conducted in Mauritania in 2015 were used for the present study. ${ }^{16}$ It was a cross-sectional survey with twostage random sampling, led by the nutrition department of the Ministry of Health with technical support from UNICEF. The survey followed SMART survey's guideline. ${ }^{17}$ All the measurements were carried out by teams of trained investigators who were experienced in taking anthropometric measurements. A national representative sample of children under 5 years old was used for this survey.

Weight was measured with a precision of $100 \mathrm{~g}$ using an electronic SECA-type weighing scale. Height was measured in centimetre with a precision of $0.1 \mathrm{~cm}$ using SHORR toises. MUAC was collected in all children aged 6-59 months with precision to $1 \mathrm{~mm}$ using MUAC tapes. UAL was measured by the same MUAC tape as those used for MUAC measurement. This length corresponds to that used to determine the mid-upper arm location, namely the length between the tip of the elbow (the olecranon) and the tip of the scapula (acromion). The oedema was systematically searched at the top of both feet by exerting a pressure with the thumb for $3 \mathrm{~s}$. Standardisation of the measurements and plausibility checks were done according to the standards and recommendations of the SMART methodology. ${ }^{17}$

\section{Data analysis}

After a double entry to clean the anthropometric data, Z-scores were calculated using Emergency Nutrition Assessment (ENA) Delta software November 2014. Children were excluded from the analysis based on the following criteria: MUAC, height, sex or weight not recorded; extreme WHZ $(<-5$ or $>+5)$; or arbitrarily considered extreme UAL $(<7 \mathrm{~cm}$ or $>30 \mathrm{~cm})$. Wasting by low WHZ was defined by (WHZ <-2) using the 2006 WHO growth reference. Wasting by low MUAC-height Z-score (MUAC-HZ) was defined by MUAC-HZ $<-2$. Wasting by MUAC-125 mm (MUAC-125) was defined by MUAC $<125$ $\mathrm{mm}$. Additionally, we compared our diagnosis approach with another MUAC cut-off proposed by Laillou and colleagues, wasting by MUAC $138 \mathrm{~mm}$ (defined by MUAC $<138 \mathrm{~mm}){ }^{18}$

Wasted children (according to the WHZ <-2) were first divided into three equal groups according to their UAL using 158 and $187 \mathrm{~mm}$ value points. To be more convenient, we arbitrarily adopted 150 and $180 \mathrm{~mm}$ as cut-off points for UAL group (UALG) classification. Thus, all children were classified in the following three groups, according to their UAL: UAL group1 (UALG1), UAL group2 (UALG2) and UAL group3 (UALG3) when UAL $\leq 150 \mathrm{~mm}$, between $151-180 \mathrm{~mm}$ and $\geq 181 \mathrm{~mm}$, 


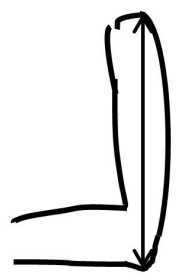

Wasting

MUAC $<125 \mathrm{~mm}$
UALG $1: \leq 150 \mathrm{~mm}$

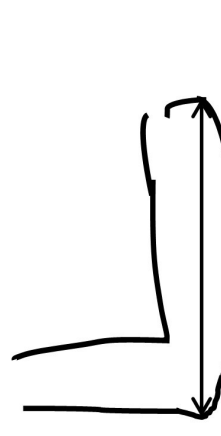

UALG $2: 151-180 \mathrm{~mm}$ Wasting MUAC $<130 \mathrm{~mm}$

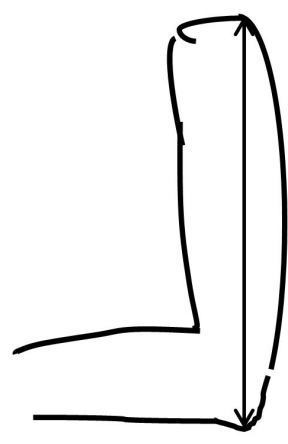

UALG $3: \geq 181 \mathrm{~mm}$ Wasting MUAC $<135 \mathrm{~mm}$
Figure 1 Classification of children according to their UAL and MUAC cut-off for each UALG. MUAC, mid-upper-arm circumference; UALG, upper arm length group.

respectively (figure 1). For each UALG, a new MUAC cut-off point was defined for wasting diagnosis. Receiver operating characteristic (ROC) methodology was used to determine new MUAC cut-offs with improved sensitivity for wasting diagnosis for each UALG with a minimum specificity of $90 \%$ (online supplemental figure 1). Data were analysed using IBM SPSS statistics software.

The accuracy of our diagnosis method was evaluated according to the Standards for Reporting of Diagnostic Accuracy Studies (STARD) recommendations. ${ }^{19}$ Wasting by $\mathrm{WHZ}<-2$ was used as reference standard to calculate the sensitivity and specificity of all the diagnosis methods that we tested. Sensitivity reflects the ability of the test to identify wasting among those identified by WHZ
$<-2$. Specificity reflects the ability to correctly identify non-wasted cases among those identified by WHZ >-2. Medcalc online version (https://www.medcalc.org/calc/ diagnostic_test.php) was used to calculate sensitivity, specificity, positive predictive value (PPV), negative predictive value (NPV), with $95 \%$ CI for each wasting diagnosis method.

\section{Statistic tests}

Mean and SD were calculated for continuous values. Correlations between continuous variables were evaluated using Pearson test. Mean UAL, MUAC, age, height and WHZ comparison among UAL groups was performed by Student's t-tests. Wasting prevalence was calculated for each wasting diagnostic method.

This analysis does not require ethical committee approval because the data are anonymous. A steering and ethics committee has been set up by the Ministry of Health and UNICEF to validate the protocol of the survey, including the ethical aspects relating to the care of severely malnourished children detected during the field survey.

\section{RESULTS}

Anthropometric measurements were taken from 12626 children aged 6-59 months throughout Mauritania. In total, 36 children $(<0.29 \%)$ presenting missing or inaccurate data were excluded from analysis (figure 2 ). A total of 12590 children with $49.9 \%$ girls were included in this

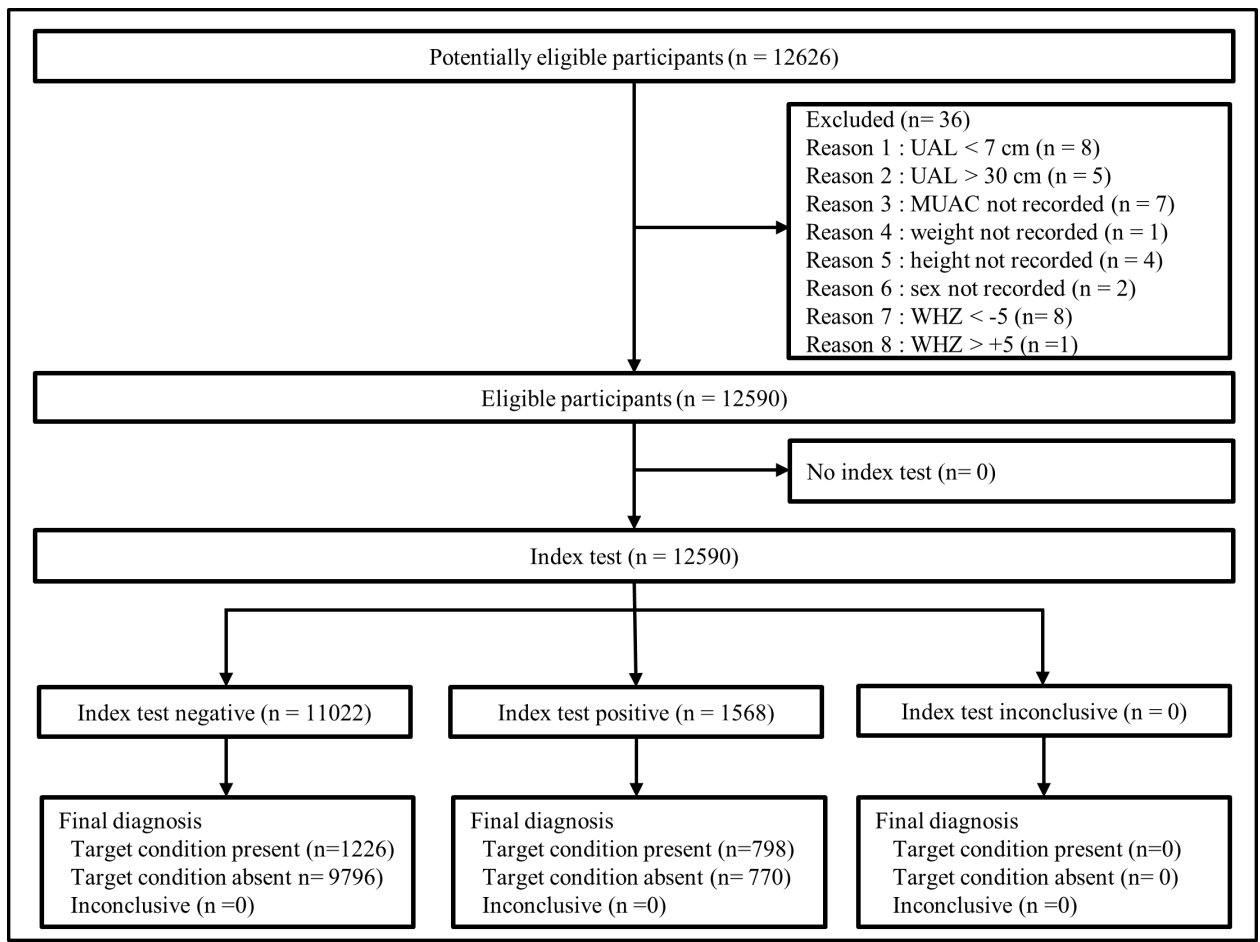

Figure 2 Flow of participants for wasting diagnosis test. Children with not recorded MUAC, weight, height or sex were excluded. Children with too high or too low UAL were excluded. MUAC, mid-upper arm circumference; UAL, upper arm length; WHZ, weight-for-height Z-score. 
Table 1 Anthropometric measurements by UALG

\begin{tabular}{|c|c|c|c|c|c|c|c|c|c|}
\hline & & Age, months & & Weight, kg & & Height, cm & & MUAC, mm & \\
\hline & $\mathbf{N}$ & Mean & SD & Mean & SD & Mean & SD & Mean & SD \\
\hline UALG1 & 2582 & $17.9^{\mathrm{a}}$ & 12.2 & $8.8^{\mathrm{a}}$ & 2.4 & $75.6^{a}$ & 10.3 & $137.3^{\mathrm{a}}$ & 12.1 \\
\hline UALG3 & 5784 & $39.4^{c}$ & 13.0 & $12.6^{c}$ & 2.4 & $92.9^{c}$ & 9.4 & $147.3^{c}$ & 11.3 \\
\hline Total & 12590 & 30.2 & 15.2 & 11.0 & 2.8 & 85.7 & 11.6 & 143.2 & 12.0 \\
\hline
\end{tabular}

UALG1 $\leq 150 \mathrm{~mm}, 151 \leq \mathrm{UALG} 2 \leq 180$, UALG3 $\geq 181 \mathrm{~mm}$. T-test was used to compare all continuous variables. Mean values that are in the same column and have different superscript letters are significatively different. The $p$ values were $<0.001$ between UALGs for all tested variables. MUAC, mid-upper-arm circumference; UALG, upper arm length group.

study. No child was found with bilateral oedema during the survey.

Our results demonstrated that UAL was correlated to height (Pearson correlation $=0.65, \mathrm{p}<0 \cdot 001$ ) and age (Pearson correlation $=0.62, \mathrm{p}<0.001$ ) and MUAC was correlated to age (Pearson correlation $=0.45, \mathrm{p}<0.001$ ) as well as height (Pearson correlation $=0.51, \mathrm{p}<0.001$ ).

Using ROC curves with WHZ as reference standard, new MUAC cut-offs (for wasting diagnosis) were determined for each UALG: $125 \mathrm{~mm}, 130 \mathrm{~mm}$ and $135 \mathrm{~mm}$ for UALG1, UALG2 and UALG3, (figure 1 and online supplemental figure 1).

The mean and SD of children's' age, weight and MUAC are described in table 1.

Mean MUAC, height and age significantly increased with UALG $(\mathrm{p}<0.001)$ (table 1$)$. The prevalence of wasting as determined by WHZ $<-2$ was $12.5 \%$ (table 2 ). When evaluated by MUAC-UALG method, wasting prevalence was $16.1 \%$. With MUAC-125, MUAC-HZ and MUAC-138, the prevalence of wasting was $5.0 \%, 6.3 \%$ and $31.7 \%$, respectively.

The diagnosis test accuracy for each indicator is summarised in the table 3. Overall, MUAC-125 had the lowest sensitivity $(17.98 \%(16.33 \% ; 19.73 \%))$ and the highest specificity $(97.49 \%$ (97.18; 97.78)) (table 3). With single fixed cut-off indicators (MUAC-125 or MUAC-138) sensitivity decreases, and specificity increases with UALG. This was not observed with adapted cut-offs (MUACHZ or MUAC-UALG) (online supplemental table 1). Although MUAC-138 had the highest sensitivity $(69.76 \%$ $(67.71 ; 71.76))$, it had the lowest specificity $(75.64 \%$ $(74.81 ; 76.45))$ leading to more than $24 \%$ false positives. MUAC-UALG had a higher sensitivity (39.43\% (37.29; 41.59)) than MUAC-125 and MUAC-HZ. MUAC-UALG had a higher specificity than MUAC-138 and a lower specificity than MUAC-HZ and MUAC-125.

MUAC-125 had a lower PPV $(57.87 \%(54.15 ; 61.50))$ than MUAC-HZ $(65.62 \%(62.43 ; 68.67))$ and a lower NPV than that of all other indicators. MUAC-138 had the lowest PPV (35.42\% (34.42; 36.44)) although the NPV was the highest among the indicators $(92.89 \%$ (92.43; 93.32)).

\section{DISCUSSION}

In this study, we demonstrated two principal results related to the use of MUAC-UALG.

First, the use of UAL along with MUAC enhanced wasting prevalence estimation (table 2). Wasting prevalence evaluated by MUAC-UALG was the closest to that of WHZ <-2 when compared with other existing diagnosis methods. Using MUAC-125 and MUAC-HZ, wasting prevalence was three and two times lower than that of WHZ $<-2$, respectively. Wasting prevalence determined by MUAC-138 was almost three times higher than that of WHZ $<-2$. Fixed cut-off MUAC often overestimates or underestimates the number of wasting cases, depending on the threshold chosen. ${ }^{9}{ }^{12}$ A fixed cut-off of $138 \mathrm{~mm}$ makes it possible to diagnose cases of wasting in older children (over 36 months), but overestimates the number of wasting cases in the youngest children (online supplemental table 2). Wasting prevalence according to the WHO standard MUAC cut-off of $125 \mathrm{~mm}$ is two times lower than that determined by the WHZ $<-2$ (table 2). When using a fixed cut-off at $138 \mathrm{~mm}$, the prevalence is two times higher than the prevalence using WHZ $<-2$. With the MUAC-UALG method, more wasted children belonging to UAL groups 2 and 3 can be diagnosed.

Table 2 Wasting prevalence determined by different methods

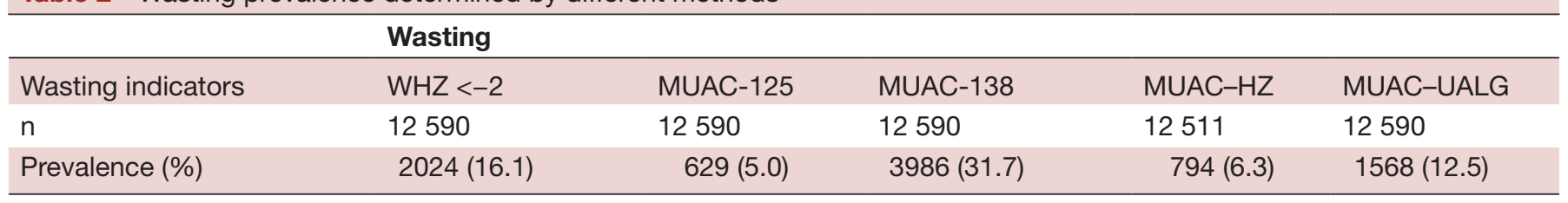

WHZ <-2, weight-for-height Z-score <-2; MUAC-125, mid-upper-arm circumference <125 mm; MUAC-138, mid-upper-arm circumference <138 mm; MUAC-HZ, MUAC-height Z-score; MUAC-UALG, mid-upper-arm circumference per upper arm length group. 
Table 3 Wasting diagnosis accuracy based on sensitivity, specificity, positive and negative predictive value for each indicator

\begin{tabular}{|c|c|c|c|c|c|c|c|c|}
\hline & \multicolumn{2}{|c|}{ Sensitivity } & \multicolumn{2}{|c|}{ Specificity } & \multicolumn{2}{|c|}{$\begin{array}{l}\text { Positive predictive } \\
\text { value }\end{array}$} & \multicolumn{2}{|c|}{$\begin{array}{l}\text { Negative predictive } \\
\text { value }\end{array}$} \\
\hline & $\%$ & $95 \% \mathrm{Cl}$ & $\%$ & $95 \% \mathrm{Cl}$ & $\%$ & $95 \% \mathrm{Cl}$ & $\%$ & $95 \% \mathrm{Cl}$ \\
\hline Wasting by MUAC-138 & 69.76 & 67.71 to 71.76 & 75.64 & 74.81 to 76.45 & 35.42 & 34.42 to 36.44 & 92.89 & 92.43 to 93.32 \\
\hline Wasting by MUAC-HZ & 26.04 & 24.13 to 28.02 & 97.40 & 97.08 to 97.70 & 65.62 & 62.43 to 68.67 & 87.37 & 87.08 to 87.65 \\
\hline
\end{tabular}

Weight-for-height Z-score $<-2$ was used as reference standard.

MUAC-125, mid-upper-arm circumference <125 mm; MUAC-138, mid-upper-arm circumference <138 mm; MUAC-HZ, MUAC-height Zscore; MUAC-UALG, mid-upper-arm circumference by upper arm length group.

Second, the use of UAL in combination with MUAC enhanced the wasting diagnosis accuracy. We selected MUAC cut-offs for each UALG in such a way to minimise the number of false positives (online supplemental figure 1). Higher sensitivity could be obtained by selecting higher MUAC cut-offs for each UALG, but we believe that this approach would have a negative impact on the malnutrition management system. Although the whole community needs nutrition interventions, those who are malnourished need it more. In our study, around $82 \%$ (1-sensitivity) of children with WHZ $<-2$ were not diagnosed with wasting when the current WHO MUAC cut-off (MUAC-125) was used (table 3). The use of this single cut-off leaves older children behind, but using a higher single cut-off is not adequate either. Indeed, an increasingly high rate of non-malnourished children could raise health workers' burden and affect the quality of wasting management. Although MUAC-UALG alone could not detect all malnourished children, the overlap between WHZ and MUAC-UALG is higher than the overlap between MUAC-125 and WHZ. MUAC-UALG method allows children's age (for non-stunted children) and height to be taken into account through their arm length, unlike with the MUAC-125 approach. Fiorentino and colleagues showed that MUAC-125 was more adapted to younger children. ${ }^{20}$ Thus, this method will allow field workers to diagnose more wasted children according to WHZ compared with the use of MUAC-125. MUAC by age group could be considered as a viable method but would not be accurate in stunted children. Moreover, children's ages are not always easy to determine in the field, whereas UAL can be measured very easily. Fiorentino and colleagues had proposed different cutoffs according to age group and sex for children under 5 years old. With their method, the sensitivity ranged between $68 \%$ and $70 \%$ but the false positive rate was high, ranging between $30 \%$ and $32 \% .{ }^{20}$ Further studies on MUAC-UALG that evaluate the link with mortality are needed. Studies investigating wasting diagnostic methods could consider the MUAC-UALG as a diagnosis mean for comparison in the future. ${ }^{21}$ Except for the MUAC-HZ for which ENA software did not provide values for 79 children, each indicator's accuracy was calculated in the same population. Thus, indicator accuracies were compared with no risk of statistical bias. The MUAC-UALG method does not require any harmful nor stressful actions against children.

The study was conducted in the Mauritanian population which is not representative of the world population. However, a multicentric study in different populations is feasible given the simplicity of collecting children's UAL. WHZ was used as a reference standard for this study although this index is only a proxy for wasting. The overlap ratio between WHZ and MUAC varies by country. ${ }^{5}$ However, WHZ is widely used and accepted for wasting prevalence estimation around the world by the WHO. A more specific wasting diagnosis tool is needed in the future to compare with MUAC-UALG. Other alternative approaches could be used to evaluate the accuracy of MUAC-UALG method to identify more vulnerable children. Thus, MUAC-UALG mortality and or morbidity prediction capacity, and its association with wasting clinical biomarkers among children with low grade inflammation status could be considered.

At the community level, compared with the WHZ method, it is easier to use the MUAC-UALG which does not require any investment in equipment to measure height and weight. Measuring height and weight can be a challenge in emergency settings such as in COVID-19 context. The portability of the MUAC tape is an advantage for its adoption by community health workers. The cost is also much lower than a scale measuring height and weight. Three MUAC tapes with different cut-offs according to UALG can be used by community health workers in the field for wasting diagnosis.

This study is aligned with the Council of Research and Technical Advice on Acute Malnutrition (CORTASAM) recommendations regarding the priority research. ${ }^{22}$ Indeed, CORTASM group has recognised that the current MUAC admissions criteria for wasting (MUAC-15mm) does not select for all high-risk children, leaving behind some children who would be diagnosed as wasted by WHZ or WAZ methods. More research is needed concerning the options available to identify these high-risk children 
and ensure successful diagnosis and treatment, but the MUAC-UALG method is a promising candidate.

To the best of our knowledge, the use of UAL in wasting diagnosis has never been proposed. This method does not add any additional tasks to the diagnostic process and has the potential to improve it. This method could be adopted in the field as a part of monitoring nutritional status of children and as an admission criterion in community-based management of acute malnutrition. Like MUAC-height or MUACage Z-score, future studies aimed at the creation of a MUAC-UAL Z-score should be considered. Using UAL-for-age Z-score could also be considered as a substitute for the height-for-age method in diagnosing cases of chronic malnutrition. Indeed, UAL is simpler and less expensive than height measurement. A comparison of each child's UAL with a same age and sex reference population could be considered for stunting diagnosis. Thus, in nutrition programmes, weight-for-age monitoring could be supplemented with UAL-for-age in cases where children's height is not known.

Beside wasting, obesity is also a major concern even in LMIC. ${ }^{4}$ Increasing the MUAC cut-off for wasting diagnosis for all children could have a negative impact if many non-wasted children are treated. It could also prevent those in need to get enough supplements in an event of shortage. Our data showed that $7.9 \%$ of children were considered as wasted despite having a WHZ $>-1$ when MUAC $<138 \mathrm{~mm}$ is used. With MUACUALG this percentage drops to $2.2 \%$.

\section{CONCLUSION}

Wasting diagnosis with a fixed cut-off MUAC has limitations that can be mitigated using MUAC-for-height and MUACfor-age indicators. The complexity of accurately collecting age and height in the field makes MUAC-UALG a good alternative for wasting diagnosis and prevalence estimation. MUAC-UALG could be used in emergency settings such as in COVID-19 context. The sensitivity is improved without compromising the specificity. Thus, using UAL along with MUAC enhances the accuracy of wasting diagnosis and the estimation of wasting prevalence. Future studies involving data from more children in different regions may lead to new perspectives on the use of MUAC-UALG as an anthropometric measure to diagnose wasting in developing countries. We recommend the inclusion of arm length in every national nutritional survey to collect more data for a multicentric study.

Acknowledgements We would like to thank all participants and all investigators for their effort in data collecting.

Contributors MDB and MB contributed in the article equally. They designed, approved and are accountable for the work, MDB more specifically conducted the field study, carried out the data editing contributed to the design of the study, the statistical analyses, the writing of the manuscript and the coordination of coauthor inputs (study design, data collection, data analysis, data interpretation, writing). MB contributed to the design of the study conducted the statistical analyses, produced the tables and graphs, reviewed the documentation, the integration of the different inputs and the writing of the manuscript (figures, study design, data collection, data analysis, data interpretation, writing). DC and NZ participated in the analysis of the results and the interpretation of the data. TB and SNB has done a complete review of the statistical analyses of the data and tables of the results. YD reviewed the documents and provided an external perspective on the concept, analysis, interpretation and conclusions of the article (revising it critically for important intellectual content)

Funding The authors have not declared a specific grant for this research from any funding agency in the public, commercial or not-for-profit sectors.

Competing interests MB works for Nutriset S.A.S, Malaunay, France. This study started before he joined the company and is independent to his activity at the company. No other author has a conflict of interest related to this study.

Patient consent for publication Not required.

Provenance and peer review Not commissioned; externally peer reviewed.

Data availability statement Data are available upon reasonable request by email to the corresponding author.

Supplemental material This content has been supplied by the author(s). It has not been vetted by BMJ Publishing Group Limited (BMJ) and may not have been peer-reviewed. Any opinions or recommendations discussed are solely those of the author(s) and are not endorsed by BMJ. BMJ disclaims all liability and responsibility arising from any reliance placed on the content. Where the content includes any translated material, BMJ does not warrant the accuracy and reliability of the translations (including but not limited to local regulations, clinical guidelines, terminology, drug names and drug dosages), and is not responsible for any error and/or omissions arising from translation and adaptation or otherwise.

Open access This is an open access article distributed in accordance with the Creative Commons Attribution Non Commercial (CC BY-NC 4.0) license, which permits others to distribute, remix, adapt, build upon this work non-commercially, and license their derivative works on different terms, provided the original work is properly cited, appropriate credit is given, any changes made indicated, and the use is non-commercial. See: http://creativecommons.org/licenses/by-nc/4.0/.

ORCID iD

Mouhamed Barro http://orcid.org/0000-0003-1731-4447

\section{REFERENCES}

1 World Health Organization,, UNICEF. WHO child growth standards and the identification of severe acute malnutrition in infants and children: a joint statement by the World Health Organization and the United Nations Children's Fund, 2009. Available: http://www.ncbi. nlm.nih.gov/books/NBK200775/ [Accessed 1 Dec 2019].

2 UNICEF. Community-based management of severe acute malnutrition: a joint statement by the World Health Organization, the World Food Programme, the United Nations System Standing Committee on Nutrition and the United Nations Children's Fund. Geneva: UNICEF, 2007.

3 Goossens S, Bekele Y, Yun O, et al. Mid-upper arm circumference based nutrition programming: evidence for a new approach in regions with high burden of acute malnutrition. PLOS One 2012;7:e49320.

4 UNICEF, WHO, World Bank Group. Levels and trends in child malnutrition: key findings of the 2020 edition of the joint child malnutrition estimates. United Nations Children's Fund, World Health Organization, World Bank Group, 2020. data.unicef.org/nutrition

5 Grellety E, Golden MH. Weight-for-height and mid-upper-arm circumference should be used independently to diagnose acute malnutrition: policy implications. BMC Nutr 2016;2.

6 Jelliffe DB. The assessment of the nutritional status of the community; with special reference to field suveys in developing regions of the world. Geneva, 1966.

7 Shakir A, Morley D. Measuring malnutrition. The Lancet 1974;303:758-9.

8 Lindtjørn B. Measuring acute malnutrition: a need to redefine cut-off points for arm circumference? The Lancet 1985;326:1229-30.

9 Bern C, Nathanail L. Is mid-upper-arm circumference a useful tool for screening in emergency settings? Lancet 1995;345:631-3.

10 Gernaat HB, Dechering WH, Voorhoeve HW. Absolute values or $\mathrm{Z}$ scores of mid-upper arm circumference to identify wasting? evaluation in a community as well as a clinical sample of under fives from Nchelenge, Zambia. J Trop Pediatr 1996;42:27-33. 
11 Hall G, Chowdhury S, Bloem M. Use of mid-upper-arm circumference $Z$ scores in nutritional assessment. The Lancet 1993;341:1481.

12 de Onis M, Habicht JP. Anthropometric reference data for international use: recommendations from a world Health organization expert Committee. Am J Clin Nutr 1996;64:650-8.

13 Janes MD, MacFarlane SBJ, Moody JB. Anthropometric measurement of malnutrition. The Lancet 1979.

14 de Onis M, Yip R, Mei Z. The development of MUAC-for-age reference data recommended by a who expert Committee. Bull World Health Organ 1997;75:11-18.

15 Mei Z, Grummer-Strawn LM, de Onis M. The development of a MUACfor-height reference, including a comparison to other nutritional status screening indicators 1997;75:9.

16 Ministère de la Santé, direction de la Santé de base et La nutrition. Enquête nutritionnelle nationale smart Mauritanie 2015.

17 Action Against Hunger, CDC Atlanta. Rapid SMART surveys for Emergencies - Guidelines 2014
18 Laillou A, Prak S, de Groot R, et al. Optimal screening of children with acute malnutrition requires a change in current who guidelines as MUAC and WHZ identify different patient groups. PLoS One 2014:9:e101159.

19 Bossuyt PM, Reitsma JB, Bruns DE, et al. Stard 2015: an updated list of essential items for reporting diagnostic accuracy studies. BMJ 2015;351:h5527.

20 Fiorentino M, Sophonneary P, Laillou A, et al. Current MUAC cut-offs to screen for acute malnutrition need to be adapted to gender and age: the example of Cambodia. PLoS One 2016;11:e0146442.

21 Girma T, Hother Nielsen A-L, Kæstel P, et al. Biochemical and anthropometric correlates of bio-electrical impedance parameters in severely malnourished children: a cross-sectional study. Clin Nutr 2018;37:701-5.

22 Briend A, Diop El, Lemma F. A research agenda for acute malnutrition. A statement from the council of researche \& technical advice on acute malnutrition (CORTASAM). Council of Research \& Technical Advice on Acute Malnutrition 2018. 